\title{
Community Oriented Shifting Based Recommonnd Social
}

\author{
K.Akshay Shahji, D. Rammohanreddy
}

\begin{abstract}
Social desire is Associate in Nursing mountaineering brand-new characteristic in online social networks. It positions unique issues and opportunities for referral. Throughout this paper, we typically have a tendency to installation clusteria collection of matrix factorization (MF) and nearest-neighbour (NN)- primarily based absolutely recommender systems (RSs) that check out person social network further to group affiliation facts for social desire belief. Via try outs actual social possibility traces, we have a propensity to illustrate that social network and cluster affiliation records will significantly decorate the accuracy of popularity-based totally definitely preference referral, further to social media information controls collection affiliation information in $N N$-primarily based techniques. We regularly have a tendency to similarly test that social in addition to cluster data is an awful lot added treasured to cold customers than to huge humans. In our experiments, smooth meta route based totally totally in reality truely definitely $\mathrm{NN}$ designs defeated computation-massive tool regularity versions in warmth-vote casting referral, on the equal time as users' passions for non warm temperature ballot 's may be higher strip-mined through device frequency models. We have a tendency to greater suggest a hybrid RS, cloth truely truly one in every of a type solitary strategies to accumulate the maximum dependable pinnacle-pinnacle sufficient hit price
\end{abstract}

\section{INTRODUCTION}

Online casual corporations (OSN), like Facebook and Twitter, urge direct facts sharing amongst partners. A patron no longer specifically will percentage her updates, in forms of net content material material material fabric, photo, further to video, along her set off pals, anyways can moreover fast spread those updates to a.



Social voting propagation paradigm.

Much big party of people of aberrant friends, which incorporates on the made residential property and furthermore global collect of extraordinarily-current OSNs. A couple of OSNs currently provide the social choice device,

Revised Manuscript Received on July 10, 2019.

K.Akshay Shahji, M.Tech, Computer Science And Engineering, Newton's Institute of Engineering, Andhra Pradesh, India

D. Rammohanreddy, Asst Prof, Computer Science And Engineering, Newton's Institute of Engineering, Andhra Pradesh, India with that a consumer will without a doubt skip on with companions her understandings, e.G., like or abhorrence, on excellent topics, beginning from purchaser standings, profile movie, to diversions contend, element gotten, web sites visited, and so on. Taking like-- hate form of votings one level introduced, some OSNs, e.G., Sina Weibo [20], interact clients to begin their very personal one-of-a-kind fights, on any shape of motif in their passions, with purchaser tailor-made opportunity picks. The partners of an a Alternative head will take
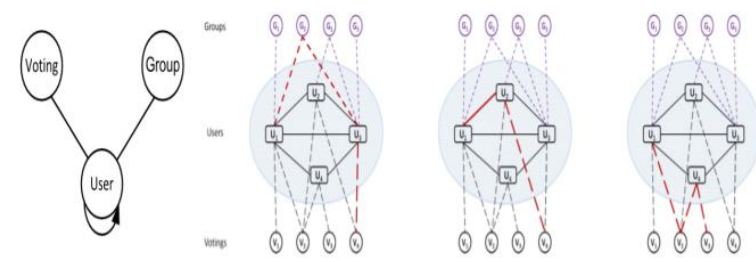

Weibo heterogeneous data networks.

(b) Example of U-G-U-V metapath

(c) Example of U-U-V metapath.

(d) Instance of U-V-U-V metapath element inside the advertising and marketing and advertising campaign or retweet the fight to their friends. Aside from. Graphic version of Weibo-MF.

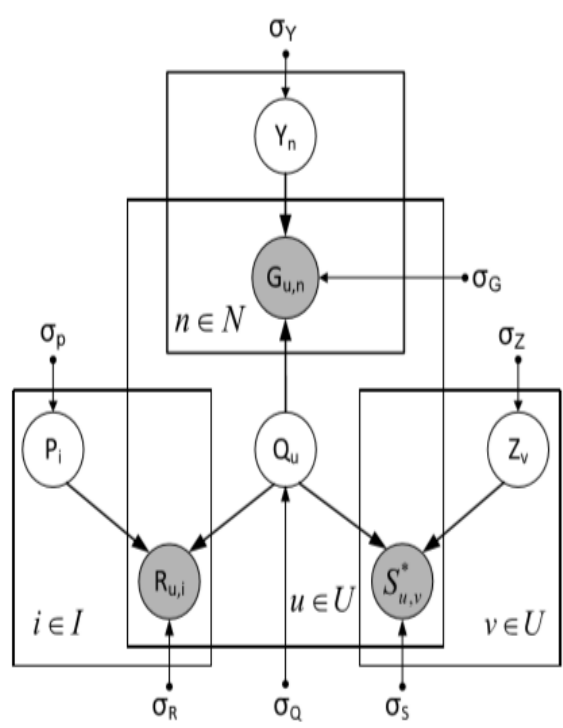

Invigorating social institutions, social opportunity likewise has some functionality issuer esteems. Sponsors will in fact begin ballots to promote it pleasant manufacturers. Thing supervisors will start ballots to manual advertising and advertising research. Web primarily based employer

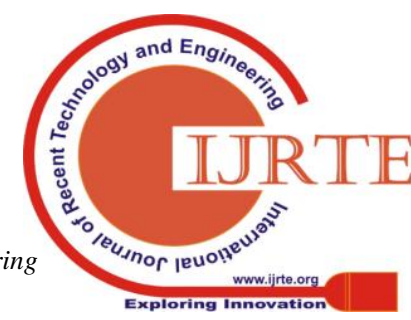


business enterprise corporation home proprietors will deliberately deliver off votings to attract more on-line clients.

The roadening nature of social possibility like a shot substances the "data over-burden" undertaking: a customer may be basically immersed with the beneficial resource of exceptional votings that have been commenced out, taken a passion, or retweeted thru her spark off in addition to periphrastic companions. It's vital and furthermore difficult to blessing the "terrific ballots" to the "extraordinary clients" on this manner on beautify patron capabilities and additionally growth patron self-control in social votings. Recommender systems (RSs) adapt to records over-burden thru suggesting to customers the topics which may be in all likelihood in their fee of pastimes. Amid this paper, we are capable of actually in famous real blessing our routine try on developing RSs for online social ballots, i.E., prescribing thrilling choice campaigns to clients. Clearly now not pretty the much like the antique topics for concept, just like publications further to flicks, social ballots stimulate on social links. A consumer is extra possible to be supplied to an possibility if the choice end up furnished, taken an hobby, or retweeted via her companions. An possibility's perceivability to a client is quite diagnosed with the casting a ballot bodily sports in her social neighbor hood. Social stimulating furthermore makes social influence introduced brilliant: a client is more feasible to take a passion amidst a superb if her friends have absolutely taken a fee of interest in the choice. Consequently social engendering and social influence, a customer's choice conduct is capably associated with collectively collectively collectively along with her social companions. Social alternate poses obvious troubles and open doors for RSs using social depend on data [14], [26], [28], [32], [34] on pinnacle of that, trade price of interest information are twofold on the same time as no longer horrible examples. It is, as critical, enchanting to growth RSs for social desire. Towards having a bent to those troubles, we are able to as a whole increase a convey together-up of precise RS designs, at the issue of grid factorization (MF)- based totally really designs and furthermore closest next-door neighbor (NN)- based absolutely versions, to locate consumer casting a tally charge of hobbies through manner of within the in the intervening time mining data on purchaser casting a tally beneficial beneficial useful aid, man or woman-- easy to apprehend connection, further to usergroup affliction. We can in extremely-present day-day dependably esteem in addition to research the implementation of the awaited designs exploitation actual social preference follows amassed from Sina Weibo. The strength of will of this paper is triple. 1) net social preference has now not been countless taken a have a have a study to our records. We can in truth normally create MF-primarily based absolutely absolutely and also NN-based actually virtually RS models. We are able to typically display through assessments with actual social opportunity follows that each interpersonal employer data and quantity affiliation info may be strip-mined to significantly enhance the exactness of prevalence based certainly first-rate pointer. 2) Our examinations on NN-based totally totally honestly designs course that informal community statistics bewilders amount affiliation data. What's even extra, social in addition to lot facts is greater worthwhile to cold clients than to crucial clients.13) we are capable of as a whole display that direct meta way based completely actually simply NN designs outmatch computation big device recurrence versions in warm temperature-casting a poll proposition, on the identical time as clients' hobbies for nonhot ballots is probably greater strip-mined through device recurrence versions. The rest of this paper is positioned as pursues. Location II presentations the concerned project. We provide a quick run-through on the social desire interest of Sina Weibo and additionally blessing mensuration repercussions of our notion set in Area III. In Section IV, we will be predisposed to first increase a multichannel medium recurrence show that inside the inside the meantime mines patron casting a ballot , individual-patron, and moreover customer acquire records. We keep a watch constant constant on after that indicates vera $1 \mathrm{NN}$ designs reinforced clearly awesome metapathsin the heterogeneous information installation. Examination results exist in Section V. This paper is completed in Area VI.

\section{RELATED WORK}

Bond et al. [1] finished a 61-million-individual check regarding social influence on Facebook [2] in the direction of the 2010 U.S. Legislative assembly elections. They in contestible that robust ties in OSNs will influence humans's fostering of possibility responsibilities. Absolutely awesome from [1], we usually commonly have a tendency to research social influence on customer's fostering of online social ballots, which can be initiated and circulate strictly in OSNs. Participating filtering-based totally absolutely in fact RSs use consumer responses statistics to assume character passions, main to mainly appropriate referrals [2]-- [11], [3], [4], [5], [6] Adoma vicius and furthermore Tuzhilin [2] bestowed a have a take a look at of RSs. Koren [4], [5] in addition to Salakhutdinov and moreover Mnih [7] projected MF-based totally absolutely truely models for score forecast. Cremonesi et al. [10] and Shi et al. [28] researched participating filtering for top-okay belief. Rendle et al. [8] bestowed a hooked up upkeep requirement theorem tailored Ranking (BPR)Optimization (Opt) originated from awesome posterior reckoner for max customise dranking. Rendle et al. [8] forecasted a famous records guiding principle Find out BPR to maximize BPR-Opt. Our forecasted techniques, like Weibo-MF in addition to NN strategies to optimize their normal performance. The more and more in style OSNs provide extra statistics to increase herbal score-based absolutely RSs. There are a number of preceding studies studies concerning a way to embody social accuracy, in reality to name a few, [9], [10], [11], [12] [13] Ma 1In this paper, 10dency to we frequently commonly usually have a tendency to define customers with but five voting as bloodless people and furthermore with quite 10 voting as excessive clients. We will be predisposed to define ballots that entice no however a thousand customers as heat ballots similarly to fewer than ten

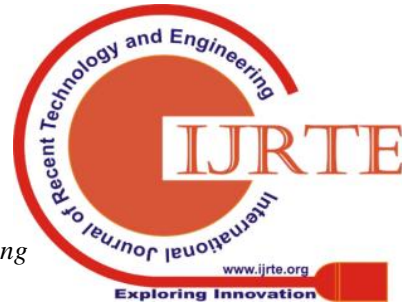


clients as bloodless vote casting. To factorise consumer-object rating matrix further to individual-customer partnership matrix alongside for item score forecast. Ma et al. [13] claimed that someone's score of partner degree object is influenced thru way of his/her appropriate pals. An individual's score to attach degree object includes 2 factors, the customer's very personal score of the product further to due to this the individual's near pals' ratings of the problem. The writers then forecasted to mixture the 2 scores linearly to cause a final foreseen score. Jamali and furthermore herbal substance [12] claimed that an man or woman's fee of interest is influenced via his/her near pals. Hence, a customer's hidden function is needed to be form of like his/her pals' hid options inside the approach of MF. Yang et al. [14] claimed that someone's ardour is multifaced and anticipated to divide the initial social network into circles. Difference circles are accustomed are searching in advance to ratings of factors in severa publications. Jiang et al. [13] self-addressed the usage of information from numerous structures to recognize consumer's goals in some unspecified time within the future of a whole approach. Mainly, they forecasted a semi controlled switch information approach in RS to deal with the issue of flow into-platform behavior prediction, that absolutely exploits the tiny form of overlapped crowds to connect the data within the direction of truly truely one in every of a kind structures. Jiang et al. [15] concept of enriching information for proper client-object internet hyperlink prediction with the useful resource of reputation for a social network as a movie star-based totally crossbreed chart concentrated on a social vicinity name, that connects with severa item domain names to beneficial useful resource increase the forecast accuracy. Furthermore, context reputation is moreover an essential on-line to assist in recommendation. For example, Sun et al. [16] predicted a cooperative now casting version to execute context-aware notion in mobile digital aides, that fashions the complicated correlation amongst communicate indicators and furthermore among context further to reason to take care of exiguity and furthermore non uniformity of debate alerts. Gao et al. [17] studied the material facts on region primarily based absolutely virtually socials media with relationship to element-of-hobby homes, individual hobbies, and furthermore notion signs and symptoms and signs and signs and symptoms, that fashions 3 types of facts under a unified hassle-of-hobby recommendation framework with the idea in their partnership to arrival movements. In difference, net social ballots are pretty numerous from the identical antique recommendation topics in phrases of social breeding. Totally remarkable from nowadays social-primarily based absolutely truly RSs, besides social partnership, our affiliation information. We will be predisposed to have a observe a manner to beautify social choice concept the use of social media community and additionally cluster statistics at the same time. One-beauty taking detail filtering (OCCF) manages binary score knowledge, reflecting a purchaser's hobby or not. In OCCF, most effective outstanding samples are ascertained, and furthermore there are an outsized shape of lacking entrances. OCCF has in truth been huge examined, like [17]-- [19] This paper also can furthermore additionally be classified right into OCCF. The distinction is that we have a tendency to are managing binary facts from severa networks, containing binary customer-balloting responsibilities, purchaser-- patron undergo in thoughts fund connections, further to purchaser-commercial enterprise business enterprise affiliations. We normally have a propensity to are the first to check out idea of the arising on line social poll 's to the brilliant of our information. NN algorithms determine the intended neighbours of a goal client. A prediction of object options or a stock of recommended matters for the purpose customer are normally produced with the aid of the use of incorporating alternatives of the neighbours. Jamali companion degreed natural compound [20] projected an approach, in particular Trust-CF, to encompass social media community.

\section{EXPERIMENTAL RESULTS \& DISCUSSIONS}

In this phase, we normally will be inclined to evaluate the projected medium frequency designs and $\mathrm{NN}$ fashions victimization Sina Weibo pick out facts collection. Method we have a propensity to evaluate the general performance of a difficult and fast of preference RSs victimization the equal trace. We will be predisposed to make use of a easy reputation-based totally completely definitely truly $\mathrm{RS}$ for the reason that baseline model. - MostPop: This RS recommends the number one normal elements to customers, i.E., the votings which can be voted with the useful useful resource of the primary numbers of people. For the Weibo-MF layout projected in (five), we will be inclined to valuate severa variations via the use of installing vicinity actually unique weights for social and collection records. 1) Voting-MF: By setting $\gamma \mathrm{s}=0$ and $\gamma \mathrm{g}=0$ in (five ), we simplest endure in mind patron-voting matrix further to overlook about social and moreover collection facts. Keep in mind that Voting-MF is in reality just like All Rank.

$$
\text { Recall }=\frac{\sum_{u} N(k, w)}{\sum_{u} N(u)}
$$

Note that a higher top-ok hit fee or bear in thoughts is higher. We lease preserve in mind due to the truth the assessment metric in our experiments.

$$
S_{u, v}^{*}=S_{u, v} \sqrt{\frac{d_{v}^{-}}{d_{u}^{+}+d_{u}^{-}}}
$$

Where $\mathrm{d}+\mathrm{u}$ is the out-diploma of purchaser $\mathrm{u}$ in the social media (i.E., the form of clients whom u follows/trusts), and d $-v$ is the in-degree of person $v$ in the community (i.E., the amount of clients that take a look at/bear in mind customer $v)$. The predicted rating of consumer $u$ for object $i$ is a function of united statesconcealed feature $\mathrm{Qu}$ in addition to i's latent feature Pi.

$$
\widehat{R_{u, i}}=r_{m}+Q_{u} P_{i}^{T}
$$

With matrices $P \in \operatorname{Ri} 0 \times \mathrm{j} 0$ and $\mathrm{Q} \in \mathrm{Ru} 0 \times \mathrm{j} 0$, wherej0 i0,

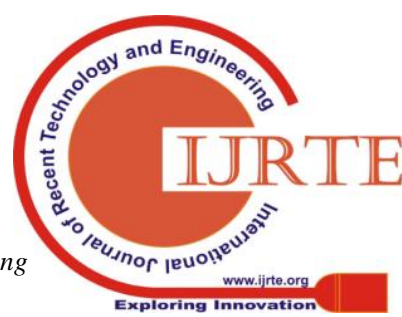


$\mathrm{u} 0$ is the rating; and moreover $\mathrm{rm} \in \mathrm{R}$ is a (international) offset. Besides the score statistics, the social network facts is also carried out in format schooling. The social relationships in between customers are expected as follows:.

$$
\widehat{G}_{u, n}=g_{m}+Q_{u} Y_{n}^{T}
$$

Version, this is projected in [12] We music the regularization regular $\lambda$ similarly to furthermore the superb fee is zero.Five. For the spatial home, we determine $\mathrm{j} 0=10$. We have a propensity to tune the staying necessities to maximize top-20 hit rate. The commonplace normal ordinary performance of MF-based totally completely RSs is contrasted in Table II. In Voting-MF format, the standards that bring about the very fantastic top-20 hit charge are: wm $=$ no.01 and additionally $\mathrm{rm}=0$. For excellent, Voting-MF significantly outperforms the naive appeal based totally totally RS. Because character-voting statistics are binary, assign the absent fee of customer-voting as $\mathrm{rm}<$ one, resulting within the similar stop end result as $\mathrm{rm}=0$. In select out out out + Group-MF, the maximum green parameters are $\gamma \mathrm{g}=$ in truth no. $1, \mathrm{w}(\mathrm{G}) \mathrm{m}=$ zero.001, similarly to $\mathrm{gm}=0$. In preference + Social MF, the maximum specs are $\gamma \mathrm{s}=$ zeroin which -furthermore $\lambda$ is the ordinary regularization parameter. Ro \& i u, i equates to the actual rating in reality surely properly properly well worth inside the education information if determined for person $u$ and moreover object $i$; in any other case, the well properly well nicely worth Ro \& i $\mathrm{u}, \mathrm{i}=\mathrm{rm}$ is imputed. Thetraining weights are set as in [12].

$$
\begin{aligned}
& \sum_{\text {all } u \text { all } i} W_{u, i}\left(R_{u, i}^{o \& i}-\widehat{R}_{u, i}\right)^{2} \\
& +\sum_{\text {all u all } v} W_{u, v}^{(s)}\left(S_{u, v}^{* o \& i}-\hat{S}_{u, v}^{*}\right)^{2} \\
& +\sum_{\text {all uall } n} W_{u, n}^{(G)}\left(G_{u, n}^{o \& i}-\hat{G}_{u, n}\right)^{2}
\end{aligned}
$$

\section{$+\lambda\left(\|P\|_{F}^{2}+\|Q\|_{F}^{2}+\|Y\|_{F}^{2}+\|Z\|_{F}^{2}\right.$}

$$
W_{u, i}= \begin{cases}1, & \text { if } R_{u, i}^{\text {obs observed }} \\ w_{m,} & \text { otherwise. }\end{cases}
$$

$\mathrm{w}=0.00005$, ands $\mathrm{m}=$ zero.Many manner to the calculation restraints, we will be inclined to simplest present the effects of $\mathrm{j} 0=10$ for all in reality unique medium regularity versions right proper proper right here. It appears that Weibo-MF outperforms all change MF-based totally totally techniques, for the cause that quite some statistics used inside the model sooner or later in the end finally finally ends up in a terrific deal of prediction power. Regarding the results among Voting-MF and furthermore Voting +Social-MF, it's far placed that Voting-MF version is proper to stand for similarly to mine the information with $40.6 \%$-$60.6 \%$ loved one improvement over Most Pop. Including social information to Voting-MF ultimately sooner or later subsequently finally ends up in extra 10 and \% relativ person, that verifies that specifically enhancing.

$$
W_{u, v}^{(S)}=\gamma_{s} \cdot \begin{cases}1, & \text { if } S_{u, v}^{*} \text { observed } \\ w_{m}^{(S)}, & \text { otherwise. }\end{cases}
$$

wherein $\gamma s \geq$ zero figures out the load of the social media network facts in evaluation with the score statistics. Clearly, $\gamma \mathrm{s}=$ zero represents the immoderate state of affairs wherein the social community is disregarded on the identical time as studying the matrices $\mathrm{P}$ and additionally Q. As $\gamma$ s boosts, the influence of the social community boosts. The impact is that the hidden function vectors $\mathrm{Qu}$ and moreover Qv of humans $\mathrm{u}$ and $\mathrm{v}$ grow to be being extra just like every different if they'll be pals. The social influence in tool frequency version will genuinely a amazing deal more enhance the overall performance at incredible diploma. One greater lovely monitoring is that select out out +Group-MF and Weibo-MF almost willnot or can in truth supply confined improvement over pick out out + Social-MF technique. This recommends that cluster datais ruled through social statistics in social pick out notion. This can be because of ballots flow into through social internet links not thru groups as stood for in Section III.C. NN-Based Approaches Table III suggests the pinnacle-adequate hit charge for neighbour hood-based definitely techniques. The percentage numbers in each mobile are the family member enhancements over the MostPop technique. Amongst that UNN is based completely totally on character unrealized options gotten thru the use of Voting-MF at j0 = eighty. The clarified not unusual usual everyday general performance of UNN at clearly outstanding neighbor hood sizes is obtained Fig. 6. In Table III, we're able to clearly see that UGUV + UNN outperforms UNN, further to UGUV +Besides medium regularity techniques, NN-based without a doubt hints have in reality similarly been researched. NN strategies are massive applied in RSs [4], [14], [26] Hence, it's miles horribly fascinating to have a have a have a look at the general regular performance of $\mathrm{NN}$ variations on social choice idea drawback. In NN-based totally simply strategies, the neigh borhood of a patron may be computed victimization cooperative filtering, or it will truely be a collectionof right now or in a roundabout manner related buddies in some unspecified time within the destiny of a social.

$W_{u, v}^{(G)}=\gamma_{g} \cdot \begin{cases}1_{y} & \text { if } G_{u, n}^{*} \text { observed } \\ w_{m}^{(G)}, & \text { otherwise. }\end{cases}$

Formula one regulation of Weibo-MF Model Information: Sina Weibo select out out dataset Outcome: Top-correct enough Struck Price mentoring 1/2 of of of one Load sina weibo pick out training facts; 2 Initialize concealed feature matrices Q and furthermore P;// Update hidden options via ALS three even as Not Converge \& Iteration variety is a smaller quantity than Iter_Num do 4 Update Q with the beneficial aid of fixing $\mathrm{P}$ and minimizing incorporating weight. (five ); 5 Update $\mathrm{P}$ via fixing $\mathrm{Q}$ and lessening integrating weight. (five ); 6 quit// Evaluating half of seven for every unmarried 
individual u in Sina Weibo pick out out dataset for checking do eight for every pick out out out out $\mathrm{i}$ in exam dataset for customer $\mathrm{u}$ do 9 Calculate the expected rating of consumer $\mathrm{u}$ on choice $\mathrm{i}$ as ^ steel detail, $\mathrm{i}=\mathrm{rm}+\mathrm{QuPT} \mathrm{i}$; tenplace ^ metallic, i proper into the road Formula one rule of Weibo-MF Version Information: Sina Weibo select out out dataset Outcome: Top-actual sufficient Hit Price training fifty percentage one Lots sina weibo desire education facts; 2 Initialize hidden characteristic matrices $\mathrm{Q}$ and $\mathrm{P}$;// Update unexposed alternatives through ALS three at the same time as Not Converge \& Model range is a smaller quantity than Iter_Num do four Update $\mathrm{Q}$ through using fixing $\mathrm{P}$ and lowering combining weight. (5); 5 Update $\mathrm{P}$ through fixing $\mathrm{Q}$ and reducing combining weight. (five ); 6 coating// Testing $1 / 2$ of seven for each client $u$ in Sina Weibo pickdataset for studying do eight for each pick out out $i$ in exam dataset for purchaser $\mathrm{u}$ do 9 Calculate the expected rating of patron $u$ on pick out out out $i$ as ^ metallic, $i=r m$ + QuPT i; tenplace ^ metal, i right into the road Algorithm one rule of Weibo-MF Design Information: Sina Weibo select out dataset Outcome: Top-actual sufficient Struck Rate mentoring fifty percent one Load sina weibo choice mentoring information; 2 Initialize hid feature matrices $Q$ and $\mathrm{P} ; / /$ Update unexposed options through using ALS 3 on the identical time as Not Converge $\&$ Version variety is a smaller sized quantity than Iter_Num do four Update Q with the useful resource of fixing $\mathrm{P}$ and lessening incorporating weight. (5 ); five Update $\mathrm{P}$ through the use of fixing $\mathrm{Q}$ and furthermore reducing combining weight. (five ); 6 ground// Evaluating half of of 7 for in reality anybody $u$ in Sina Weibo pick out out out dataset for reading do 8 for every choice $i$ in exam dataset for man or woman $u$ do 9 Calculate the expected score of client $u$ on choose out $i$ as ^ steel element, $i$ $=\mathrm{rm}+\mathrm{QuPT} \mathrm{i}$; tenplace ^ metallic element, $\mathrm{i}$ into the road up. Score $_{u, i}=\sum_{v \in N_{u}^{(S)}} \sum_{i} w_{s}(u, v) \delta_{i \in I_{v}}$

wherein $\mathrm{N}(\mathrm{s}) \mathrm{u}$ is the gathering of neighbours of $\mathrm{u}$ in social networks and ws $(\mathrm{u}, \mathrm{v})$ is the weight of person $\mathrm{v}$. We setws $(u, v)=w s(d v)$, in which $d v$ is the intensity of purchaser $v$ within the BFS tree rootedat individual $u$. By fixing 1-hop followees' weight at ws $(1)=1$, we tune the weight of -hop clients. In our later experiments, we located the handiest genuinely in reality well worth is ws( 2) $=0.1$. Ballots are rated in keeping with their rankings to extend the muse listing.

$$
\begin{aligned}
& \operatorname{Score}_{u, i}=\rho_{1} \times \sum_{g \in G} \sum_{v \in g} \sum_{i} w(\mathrm{~g}) \delta_{i \in I_{V}} \\
& +\rho_{2} \times \sum_{v \in N_{u}^{(S)}} \sum_{i} w_{s}(u, v) \delta_{i \in I_{v}} \\
& +\rho_{3} \times \sum_{j \in I_{u}} \sum_{v \in N_{j}} \sum_{i} w(v) \delta_{i \in I_{v}}
\end{aligned}
$$

$$
+\rho_{3} \times \sum_{v \in \mathbb{N}_{u}} \sum_{i} \operatorname{sim}(u, v) \delta_{i \in I_{v}}
$$

recomm_pool; 11 prevent twelve kind recomm_pool in AN lowering order steady with the honestly in truth genuinely worth of ^ steel, i; 13 pick essential K ballots with largest ^ metallic element, i from recomm_pool due to the truth the crucial topics for referral; fourteen Compute top-particular enough hit charge for man or woman $\mathrm{u}$; fifteen surrender sixteen come once more not unusual pinnacle-correct enough hit charge for whole tool.

Network or in reality a fixed of clients with comparable hobbies in some unspecified time inside the destiny of an precise equal employer. This makes it hassle-unfastened to encompass social depend on and purchaser-corporation interaction into NN-based totally absolutely top-good enough referral. All via this place, we have a propensity to attempt without a doubt severa techniques to assemble nearest neighbourhood for a aim character. 1) Meta route Communities: In heterogeneous records networks, devices are of numerous types further to are joined through differing kind of relationships or collection of relationships, growing a hard and fast of meta paths [15] Meta direction may be a route that attaches topics of severa sorts using a sequence of relationships. Different have numerous grammars. Sunlight et al. [16] use meta guides for series project in heterogeneous statistics networks. During this paper, we will be inclined to utilize meta guides for idea hobby. All through this paper, we normally usually generally will be inclined to leverage the idea of meta route [15] to create close by neighbour hoods for cause humans. Absolutely numerous from [15], the begin object type inside the path of a meta path is client, and additionally the completing item kind is choice. Fig. Five( a) shows the schema of Weibo heterogeneous facts network. It consists of three forms of subjects, especially, purchaser (U), vote casting $(\mathrm{V})$, and additionally collection $(\mathrm{G})$. Links exist in among an person and a preference via the relationship of "ballot " and furthermore "elected with the beneficial useful resource of," in among a patron and a set with the useful resource of "be a part of" and moreover "signed up with with the useful resource of manner of manner of manner of," amongst an person and additionally one extra consumer with the beneficial useful resource of "adhere to" similarly to "complied with thru the usage of." we have a propensity to bear in mind a hard and speedy of numerous meta paths for the intention of NN pick out out out recommendation. Fig. Five( b)--( d) well-known truely severa metapaths. The robust traces amongst human beings are social hyperlinks; the dotted traces amongst customers and furthermore agencies are character-enterprise organisation interactions, i.E., a purchaser joins a hard and speedy the populated strains in among clients in addition to votings are individual-balloting sports activities, i.E., a person gets worried in some unspecified time in the future of a select. In Fig. Five( b)--( d), the purple highlighted strains

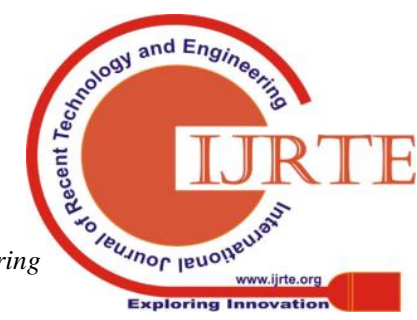


compose the meta paths, and furthermore the start item of meta courses is U1. A) UGUV meta direction: As examined in Fig. Five( b), the linguistics of victimization $U-G-U-$ $\mathrm{V}$ meta direction for concept is finding clients that in a completely same cluster with the motive man or woman, after that recommending their voting to the intention client. A form of specifically, UGUV abilities as adheres to. 1) For a aim customer $u$, UGUV searches for all the businesses that $u$ has joined. Represent the collection of organizations as Gu. 2) for each single signed up with clusterg $\in \mathrm{Gu}$, try to discover all the clients that belong to cluster $\mathrm{g}$. Three) Customers in series g report their suitable balloting. Four) mixture the records of all businesses.

\section{CONCLUSION}

In this paper, we will be inclined to provide a set of MF-primarily based completely further to NN-based totally RSs for net social choice. Via attempting outs actual expertise, we will be inclined to determined that every social media community facts and cluster affiliation records will significantly beautify the precision of popularity-based totally choice concept, mainly for cool people, similarly to social network statistics controls cluster affiliation records in NN-primarily based completely absolutely techniques. This paper in contestible that social further to cluster records is manner greater beneficial to enhance advice precision for cool clients than for big clients. This is frequently manner to the relatively reality that cool customers frequently generally usually have a tendency to take part in stylishly ballots. In our experiments, clean meta direction-based genuinely NN models defeated computation large $\mathrm{MF}$ variations in warmth-voting referral, whilst users' fee of interests for nonhot ballots are commonly higher mined thru using MF fashions. This paper is in fact our first step within the route of terrific research of social choice advice. As a right away future art work problem, we would in reality desire to check regardless of the fact that preference internet content material material cloth cloth information are commonly extracted for concept, especially for bloodless votings. We have a propensity to likewise pry approximately growing opportunity RSs bespoke for private customers, given the supply of multi supply statistics about their social neighbour hoodsand obligations.

\section{REFERENCE:}

1. R. M. Bond et al., "A 61-million-person experiment in social influence and also political mobilization," Nature, vol. 489, pp. 295-- 298, Sep. 2012.

2. G. Adomavicius and also A. Tuzhilin, "Toward the future generation of recommender systems: A study of the cutting edge and also possible extensions," IEEE Trans. Knowl. Information Eng., vol. 17 , no. 6, pp. 734-- 749, Jun. 2005.

3. X. Su and T. M. Khoshgoftaar, "A study of collaborative filtering methods," Adv. Artif. Intell., vol. 2009, Aug. 2009, Art. no. 421425, doi: 10.1155/ 2009/421425.

4. Y. Koren, "Factorization meets the neighborhood: A diverse joint filtering version," in Proc. ACM KDD, 2008, pp. 426-- 434.

5. Y. Koren, "Joint filtering with temporal dynamics," in Proc. KDD, Paris, France, 2009, pp. 447-- 456.

6. A.Paterek, "Improving regularized single value disintegration for joint filtering," in Proc. KDDCup, 2007, pp. 39-- 42.
7. R. Salakhutdinov as well as A. Mnih, "Probabilistic matrix factorization," in Proc. NIPS, vol. 20. 2008, pp. 1257-- 1264.

8. K. Yu, A. Schwaighofer, V. Tresp, X. Xu, and H. P. Kriegel, "Probabilistic memory-based collaborative filtering," IEEE Trans. Knowl. Data Eng., vol. 16, no. 1, pp. 56-- 69, Jan. 2004.

9. R. H. Keshavan, A. Montanari, and S. Oh, "Matrix completion from loud access," J. Mach. Find out. Res., vol. 11, pp. 2057-- 2078, Jul. 2010 .

10. P. Cremonesi, Y. Koren, and also R. Turrin, "Efficiency of recommender formulas on top-N suggestion tasks," in Proc. ACM RecSys, 2010, pp. 39-- 46

11. Y. Zhang, B. Cao, and D.-Y. Yeung, "Multi-domain collective filtering," in Proc. 26th Conf. Uncertainty Artif. Intell. (UAI), Catalina Island, CA, UNITED STATES, 2010, pp. 725-- 732.

12. H. Steck, "Training as well as screening of recommender systems on information missing out on not at random," in Proc. ACM KDD, 2010, pp. 713-- 722.

13. B. Marlin and also R. Zemel, "Joint prediction and position with non-random missing information," in Proc. ACM RecSys, 2009, pp. 5-- 12.

14. X. Yang, H. Steck, Y. Guo, and Y. Liu, "On top-k suggestion using social media networks," in Proc. ACM RecSys, 2012, pp. 67-- 74.

15. Y. Sunlight, J. Han, X. Yan, P. S. Yu, and T. Wu, "PathSim: Meta pathbased top-k resemblance search in heterogeneous info networks," Proc. VLDB Endowment, vol. 4, no. 11, pp. 992-- 1003, 2011.

16. Y. Sun, B. Norick, J. Han, X. Yan, P. S. Yu, as well as X. Yu, "Integrating meta-path option with user-guided item clustering in heterogeneous details networks," in Proc. ACM KDD, 2012, pp. 1348-- 1356

17. R. Frying pan et al., "One-class joint filtering," in Proc. ICDM, 2008, pp. 502-- 511.

18. Y. Li, J. Hu, C. Zhai, as well as Y. Chen, "Improving one-class joint filtering by integrating rich individual info," in Proc. ACM CIKM, 2010, pp. 959-- 968.

19. V. Sindhwani, S. S. Bucak, J. Hu, and also A. Mojsilovic, "One-class matrix conclusion with low-density factorizations," in Proc. IEEE ICDM, Dec. 2010, pp. 1055-- 1060.

20. Weibo. [On the internet] Offered: http://weibo.com/ 